\title{
Don't overlook flank pain in apparently asymptomatic COVID-19 cases
}

\author{
A case report and literature review
}

Shaden S. Al Mousa, MBBS, Ammar Ashraf, MBBS, EDiR, Abmed M. Abdelrahman, Msc, EDiR.

\begin{abstract}
بدأ الإِبلاغ عن تفشي مرض فيروس كورونا الجميد 2019 2019م (COVID-19)

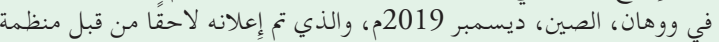

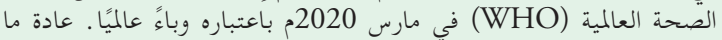

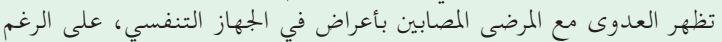

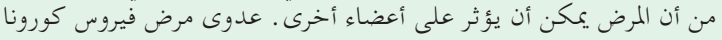

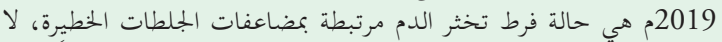

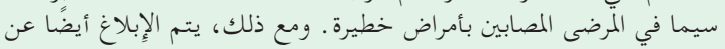

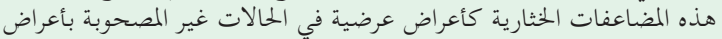

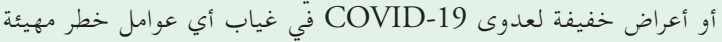

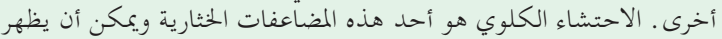

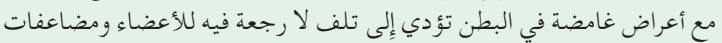

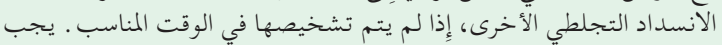

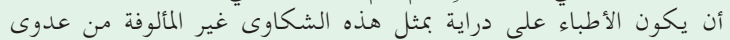

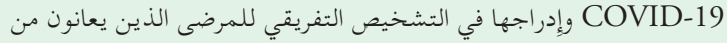

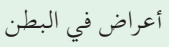

An outbreak of novel coronavirus disease-2019 (COVID-19) was reported in Wuhan, China, in December 2019, which was later declared a global pandemic by the World Health Organization (WHO) in March 2020. It is a life-threatening contagious infection with infected patients usually presenting with respiratory tract symptoms, although the disease can affect other multiple organs. Coronavirus disease-2019 infection is a hypercoagulable state associated with serious thrombotic complications, particularly in critically ill patients. However, these thrombotic complications are also being reported as a presenting symptom in asymptomatic and mildly symptomatic cases of COVID-19 infection in the absence of any other predisposing risk factors. Renal infarction is one of these thrombotic complications and can present with ambiguous abdominal symptoms leading to irreversible organ damage and other thromboembolic complications, if not diagnosed in time. Physicians must be aware of such uncommon presenting complaints of COVID-19 infection and include it in the differential diagnosis of patients presenting with abdominal symptoms.

Keywords: hypercoagulopathy, renal infarction, case report, SARS-CoV-2, COVID-19
Saudi Med J 2022; Vol. 43 (3): 307-312

doi: 10.15537/smj.2022.43.3.20210731

From the Department of Radiology (Al Mousa); College of Medicine; King Faisal University, and from the Department of Medical Imaging (Ashraf, Abdelrahman); King Abdulaziz National Guard Hospital, Al-Ahsa, Kingdom of Saudi Arabia.

Received 10th November 2021. Accepted 2nd February 2022.

Address correspondence and reprint request to: Dr. Shaden S. Al Mousa, Department of Radiology, College of Medicine, King Faisal University, Al-Ahsa, Kingdom of Saudi Arabia. E-mail: shaden.almousa@gmail.com

ORCID ID: https://orcid.org/0000-0002-8421-6196

Coronavirus disease-2019 (COVID-19) is a $\checkmark$ pandemic, lethal communicable infection that is caused by severe acute respiratory syndrome coronavirus-2 (SARS-CoV-2), which was discovered in December 2019, Wuhan, China. ${ }^{1,2}$ The target organs of this pandemic disease are the respiratory, coagulation, and immune systems. ${ }^{2-6}$ Coronavirus disease-2019 patients present with acute lung injury, which can progress to respiratory failure, although multiple organ failure can also occur. ${ }^{1,5}$

Coronavirus disease-2019 patients can also present with abdominal symptoms. As per recent data from Wuhan, 79\% of COVID-19 infected patients presented with gastrointestinal symptoms, such as diarrhea. ${ }^{6}$ The most common abdominal imaging findings described in the literature were bowel abnormalities and cholestasis. ${ }^{6}$ In addition to the gastrointestinal findings, renal abnormalities, such as renal infarction, have also been described, particularly in severely affected patients. ${ }^{3,4,6}$ Here, we present a young male patient with mild/ asymptomatic COVID-19 infection presented with flank pain secondary to the renal infarction, which was detected on the computed tomography (CT) of the abdomen. 
Case Report. A 31-year-old asymptomatic male had a history of exposure to a COVID-19-positive colleague in his office. Upon screening, his nasal swab was positive for COVID-19. He was advised home quarantine/isolation for 10 days as he was asymptomatic and vitally stable. Two weeks later, he presented to King Abdulaziz National Guard hospital's Emergency Department, Al-Ahsa, Saudi Arabia, with acute severe left flank pain (pain score: 8/10) and mild shortness of breath for the prior one day. Abdominal pain was intermittent in nature and was associated with nausea, whereas shortness of breath was associated with occasional cough. There was no history of fever, dehydration, vomiting, or change in urinary/bowel habits.

Clinical findings. Upon clinical examination, he had mild tenderness in the left lumbar region. He was vitally stable (blood pressure: $121 / 78 \mathrm{~mm} \mathrm{Hg}$, heart rate: $89 /$ minute, respiratory rate: $20 /$ minute, body temperature: $36.7^{\circ} \mathrm{C}$, and $\mathrm{SpO}_{2}=98 \%$ at room air).

Diagnostic assessment. Initial laboratory investigations showed elevated inflammatory markers (C-reactive protein, ferritin, and lactate dehydrogenase), mild leukocytosis, and mildly deranged D-dimer, prothrombin time (PT), and international normalization ratio (INR) (Table 1). His nasal swab for COVID-19 was repeated, which was still positive. Renal functions (creatinine, estimated glomerular filtration rate $[e G F R]$ ) were normal. No proteinuria was observed in the urine analysis. Thrombophilia work up was negative for antiphospholipid antibodies. The lupus anticoagulant panel was also negative. The laboratory investigations were also negative for sickle hemoglobin and paroxysmal nocturnal hemoglobinuria $(\mathrm{PNH})$. The past medical history revealed hypothyroidism (treated with levothyroxine at $150 \mathrm{mcg}$ OD) and dyslipidemia (treated with atorvastatin at $40 \mathrm{mg}$ OD). There was no personal or family history of hematological diseases. Upon cardiac evaluation, the 24-hour Holter showed sinus tachycardia (maximum heart rate: 148, minimum heart rate: 47, average: 90) with rare premature atrial contractions and premature ventricular contractions, whereas no intra-cardiac shunt was observed on the echocardiogram (transthoracic as well as transesophageal with saline bubbles). Computed tomography of the abdomen (Figure 1) showed multiple infarctions

Disclosure. Authors have no conflict of interests, and the work was not supported or funded by any drug company.
Table 1 - Laboratory investigations

\begin{tabular}{|c|c|c|}
\hline Laboratory findings & Value & Reference range \\
\hline CRP & $19.60 \mathrm{mg} / \mathrm{L}$ & $\leq 1.20 \mathrm{mg} / \mathrm{L}$ \\
\hline Ferritin & $471.7 \mu \mathrm{g} / \mathrm{L}$ & $21.8-274.6 \mu \mathrm{g} / \mathrm{L}$ \\
\hline $\mathrm{LDH}$ & $1210 \mathrm{U} / \mathrm{L}$ & $125-220 \mathrm{U} / \mathrm{L}$ \\
\hline Creatinine & $72 \mu \mathrm{mol} / \mathrm{L}$ & $64-110 \mu \mathrm{mol} / \mathrm{L}$ \\
\hline eGFR & $117 \mathrm{~mL} / \mathrm{min} / 1.73 \mathrm{~m}^{2}$ & $\geq 60 \mathrm{~mL} / \mathrm{min} / 1.73 \mathrm{~m}^{2}$ \\
\hline PTT & $27.6 s$ & $21.8-29.4 \mathrm{~s}$ \\
\hline PT & $16.5 \mathrm{~s}$ & $7.6-10.4 \mathrm{~s}$ \\
\hline PT control & $10.0 \mathrm{~s}$ & $11.0-14.0 \mathrm{~s}$ \\
\hline PT-Sec & $16.0 \mathrm{~s}$ & $11.6-14.6 \mathrm{~s}$ \\
\hline INR & 1.8 & $0.8-1.2$ \\
\hline PT-INR & 1.3 & * \\
\hline D-dimer & $0.80 \mathrm{mg} / \mathrm{L}$ & $0.00-0.50 \mathrm{mg} / \mathrm{L}$ \\
\hline WBCs & $12.20 \times 10^{9} / \mathrm{L}$ & $4-11 \times 10^{9} / \mathrm{L}$ \\
\hline Neutrophils & $8.87 \times 10^{9} / \mathrm{L}$ & $2-7.5 \times 10^{9} / \mathrm{L}$ \\
\hline Monocytes & $1.35 \times 10^{9} / \mathrm{L}$ & $0.1-1.1 \times 10^{9} / \mathrm{L}$ \\
\hline $\mathrm{RBCs}$ & $6.30 \times 10^{12} / \mathrm{L}$ & $4.5-6.1 \times 10^{12} / \mathrm{L}$ \\
\hline $\mathrm{Hgb}$ & $169 \mathrm{~g} / \mathrm{L}$ & $135-180 \mathrm{~g} / \mathrm{L}$ \\
\hline Platelets & $351 \times 10^{3} / \mathrm{mcL}$ & $150-400 \times 10^{3} / \mathrm{mcL}$ \\
\hline HCT & $0.544 \mathrm{~L} / \mathrm{L}$ & $0.42-0.54 \mathrm{~L} / \mathrm{L}$ \\
\hline Free T4 & $8.7 \mathrm{pmol} / \mathrm{L}$ & $9.0-19.0 \mathrm{pmol} / \mathrm{L}$ \\
\hline TSH & $6.78 \mathrm{mIU} / \mathrm{L}$ & $0.35-4.94 \mathrm{mIU} / \mathrm{L}$ \\
\hline Serum BGP1 IgM & 4.51 SMU & $\begin{array}{c}<20 \text { SMU }(-) \text { and }>20 \\
\text { SMU (+) }\end{array}$ \\
\hline Serum BGP1 IgG & $0.60 \mathrm{SGU}$ & $\begin{array}{c}<20 \text { SGU }(-) \text { and }>20 \\
\text { SGU (+) }\end{array}$ \\
\hline Serum BGP1 IgA & $2.11 \mathrm{SAU}$ & $\begin{array}{c}<20 \text { SAU }(-) \text { and }>20 \\
\text { SAU }(+)\end{array}$ \\
\hline ACA total Ab IgA & 2.86 APL unit & $\begin{array}{l}<12 \text { APL unit }(-),>80 \\
\text { APL unit strong }(+)\end{array}$ \\
\hline ACA total Ab IgM & 18.55 MPL unit & $\begin{array}{l}<12.5 \text { MPL unit }(-),>80 \\
\text { MPL unit strong }(+)\end{array}$ \\
\hline ACA total Ab IgG & 4.76 GPL unit & $\begin{array}{l}<15 \text { GPL unit }(-),>80 \\
\text { GPL unit strong }(+)\end{array}$ \\
\hline LA1 & $41.7 \mathrm{~s}$ & $30-43 \mathrm{~s}$ \\
\hline $\begin{array}{l}\text { Lupus group } \\
\text { anticoag }\end{array}$ & Negative & \\
\hline
\end{tabular}

Factor $\mathrm{V}$ gene

mutation (Leiden mutation)

Prothrombin (factor

II) mutation

Molecular genetic

analysis of mutation c. $1849 G>T$

p. $(V 617 F)$ in exon 14

Wild type ("Leiden" mutation not detectable)

Wild type (no mutation present)

Not detected

of the JAK2 gene

${ }^{*}$ No treatment: $>3.0$, for patient on coumadin/warfarin therapy: $>5.0$, for thromboembolic states: $2.0-3.0$, for artificial heart valves and recurrent embolism: 2.5-3.5, CRP: C-reactive protein,

LDH: lactate dehydrogenase, eGFR: estimated glomerular filtration rate, PTT: partial thromboplastin time, PT: prothrombin time,

INR: international normalization ratio, WBCs: white blood cells, RBCs: red blood cells, Hgb: hemoglobin, HCT: hematocrit,

T4: thyroxine 4, TSH: thyroid stimulating hormone, BGP1: beta

2-glycoprotein 1, ACA total Ab: anti-cardiolipin antibody, LA1: Lupus group, s: second, SAU: surgical assessment unit, Ig: immunoglobulin, APL: IgA phospholipia units 
at the mid and lower poles of the left kidney with patent main renal vessels and subpleural patchy ground glass, and reticular opacities in the scanned lung bases, which were consistent with COVID-19 pneumonia.

Therapeutic intervention. He was managed as an inpatient with therapeutic enoxaparin (low molecular weight heparin [LMWH]) $90 \mathrm{mg}$ subcutaneously for 5 days, which was later replaced with oral warfarin $6.5 \mathrm{mg}$ (to be continued for 6 months) at the time of discharge with a target INR of 2-3. The flank pain was managed with injectable acetaminophen. No specific treatment was given for the COVID-19-related pulmonary findings. He did not require intubation, intensive care unit (ICU) admission, or supplementary oxygen during his stay in the hospital.

Follow-up and outcomes. Follow up CT abdomen after 6 months showed focal scaring at the lower pole of the left kidney (Figure 2). On the follow up visits, because of the patient's poor compliance to the medical treatment with sub-therapeutic INR on multiple occasions (below 2) and higher chances of recurrence, patient was advised to continue warfarin for life-long. The key clinical findings and follow up for this patient are summarized in Figure 3.

Patient perspective. The patient tolerated well the anticoagulation and did not report any complication until now.

Discussion. Abdominal imaging, such as a CT scan, is not a routine investigation in COVID-19 patients and is carried out only in cases with abdominal pain or suspected abdominal sepsis. ${ }^{6}$ In COVID-19 patients with abdominal complaints, $16-79 \%$ cases are related to the gastrointestinal system, and the most common reported imaging findings were bowel wall thickening and cholestasis. ${ }^{6}$ Renal pathologies,
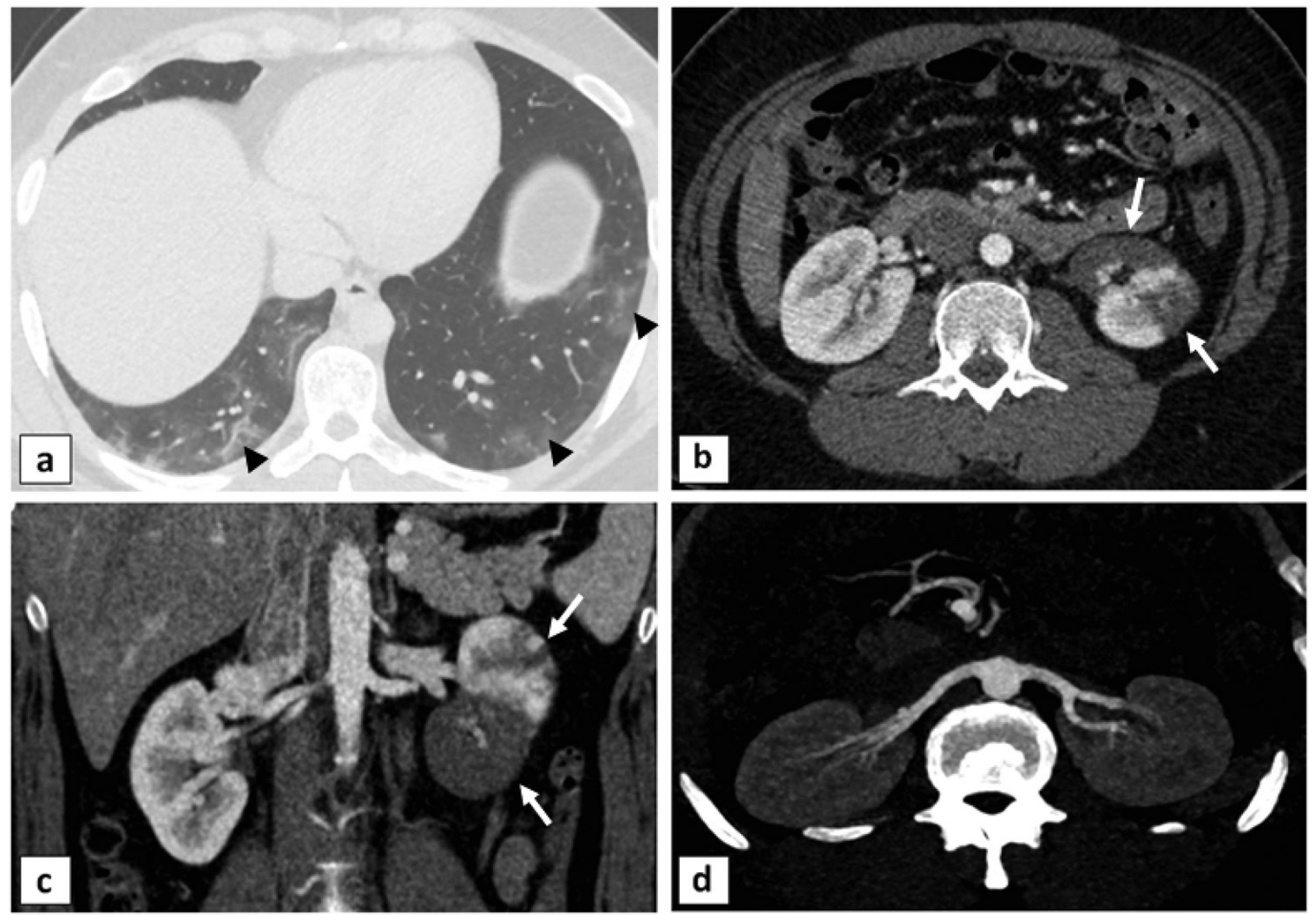

Figure 1 - Baseline axial computed tomography image through the lower chest showing: a) patchy ground glass and reticular opacities in the peripheral lung bases, b) multiple focal non-enhancing hypodensities, which are consistent with infarcts, are visible at the mid and lower poles of the left kidney on the axial and c) coronal images, and d) patent renal arteries on both sides are demonstrated by maximum intensity projection images. 

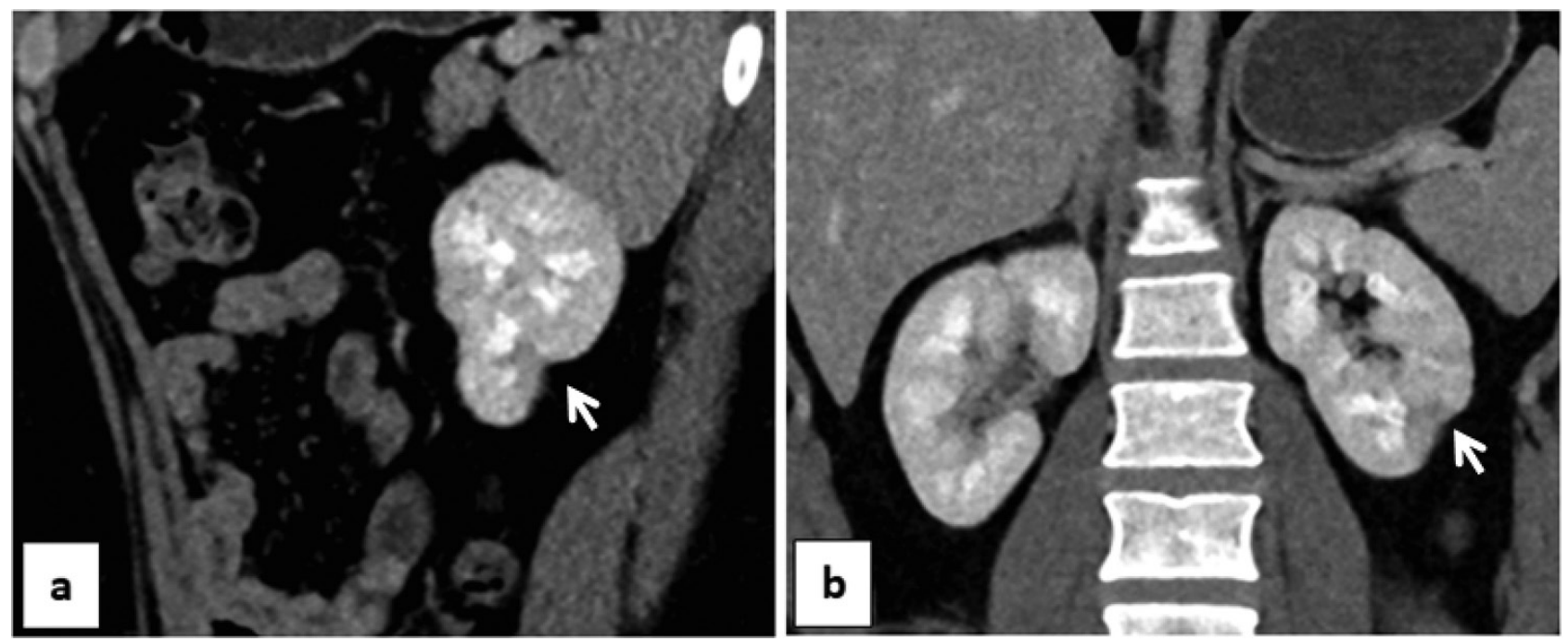

Figure 2 - A follow up computed tomography showing focal scaring at the lower pole of the left kidney. a) Sagittal and b) coronal images.

\section{1-year-old male patient}
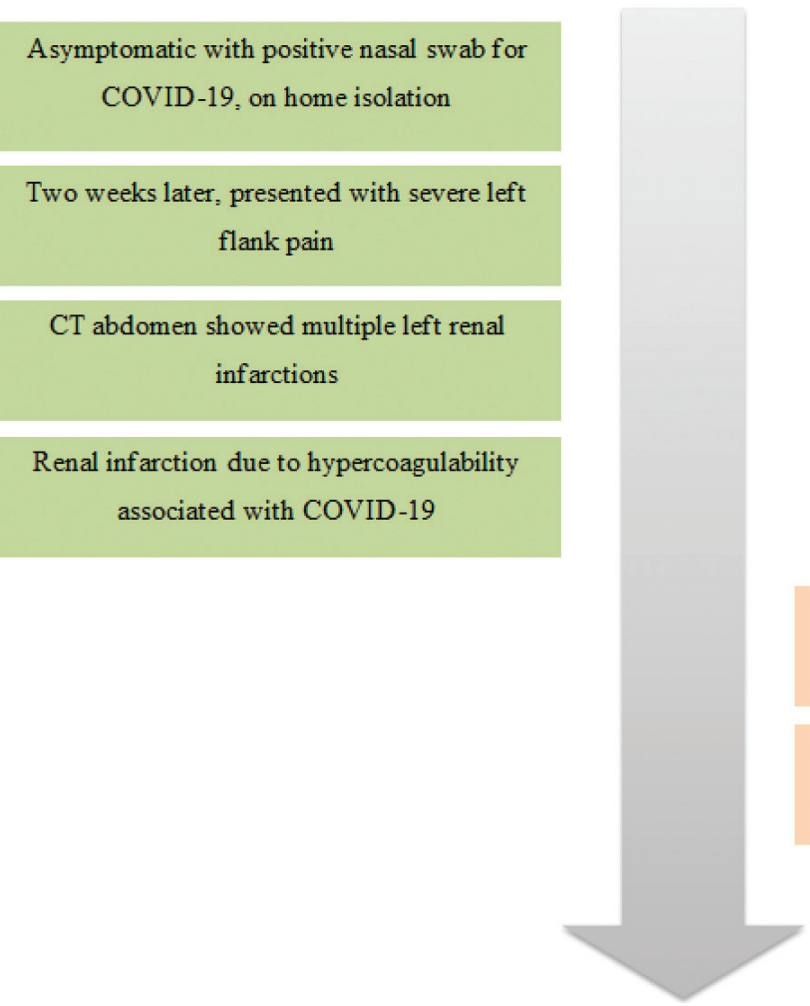

Patient was started on anticoagulation therapy

Patient was followed up regularly and improved clinically

Figure 3 - Case timeline. COVID-19: coronavirus disease-2019, CT: computed tomography 
such as COVID-19-associated acute kidney injury and a few cases of renal infarctions secondary to the hypercoagulability have also been described in such patients. ${ }^{1,3,4,6}$ Multiple centers have also described new onset proteinuria. ${ }^{3}$

According to current data, chief presenting complaints of COVID-19 were predominantly related to the respiratory system. However, it is well known to have a quite variable clinical presentation because of its ability to affect multiple other systems. ${ }^{7}$ Coronavirus disease-2019 has a diverse clinical presentation, which can vary from asymptomatic infection, self-limited influenza-type symptoms, and acute pneumonia to fatal severe respiratory failure. ${ }^{3}$ Fever $(88.7 \%)$, cough $(67.8 \%)$, fatigue $(38.1 \%)$, sputum production $(33.7 \%)$, shortness of breath $(18.7 \%)$, and myalgia $(14.9 \%)$ were the most common clinical features of COVID-19 disease, whereas nausea/vomiting (5\%) and diarrhea (3.8\%) were uncommon clinical features. ${ }^{2-6,8}$ Patients with renal infarction usually present with abdominal or flank pain, nausea/vomiting, hematuria, fever, acute renal failure, or uncontrolled hypertension. ${ }^{2}$

Hypercoagulability is one of the eminent effects of COVID-19 infection that can affect both venous or arterial systems, leading to different complications, such as deep vein thrombosis, pulmonary embolism, limb ischemia, and even ischemic strokes. ${ }^{6-8}$ These thrombotic complications were more commonly observed in severely infected patients $(79 \%$ in ICU patients versus $1.1 \%$ in non-ICU patients). ${ }^{7}$ Other studies showed thrombotic complications in approximately one-third of seriously affected COVID-19 patients. ${ }^{8,9}$

Literature review showed severe hypercoagulability with significantly high plasma D-dimer and fibrinogen levels, which correlated with worse prognosis, in severely infected COVID-19 patients. $^{5-8}$

Coronavirus disease-2019 is associated with a hypercoagulable state, and the suggested mechanisms of this coagulopathy are disseminated intravascular coagulation and endotheliopathy. ${ }^{5,10}$ Its infection activates a profound systemic inflammatory response, which generates thrombin, fibrin, and other coagulation factors. ${ }^{10}$ This initial COVID-19-associated coagulopathy (CAC) manifests with higher levels of D-dimer and fibrin/fibrinogen degradation products, whereas no significant abnormalities have been observed in the PT, partial thromboplastin time, or platelet counts. ${ }^{2,5}$ Later on, this coagulopathy is further aggravated by the involvement of the complement pathway and cytokines, such as interleukin-6 (IL-6). ${ }^{5}$ Coronavirus disease-2019 might infect endothelial cells via angiotensin converting enzyme 2 (ACE2) receptors and cluster of differentiation 13, leading to disseminated endothelial inflammation, micro vascular prothrombotic effects, and endothelial cell apoptosis, which eventually causes widespread thrombosis., ${ }^{2,510}$ This COVID-19-associated coagulopathy (CAC) may be further worsened by a decreased antithrombin production due to an underlying viral hepatic disease. ${ }^{2}$ Infrequently, due to unknown reasons, the inflammatory response is so intense that it causes uncontrolled release of mediators, such as IL-6 ("cytokine storm"), further aggravating the situation. ${ }^{2}$ Antiphospholipid antibodies have also been found in some COVID-19 patients, which may also be responsible for thrombotic events similar to antiphospholipid syndrome. . $^{1,2,4,6,8,10}$ Organ crosstalk between the affected lung, the kidney, and the heart can further worsen the situation. ${ }^{10}$

In a recent post-mortem histopathological analysis of kidneys of 26 patients with severe COVID-19 infection, $\mathrm{Su}$ et $\mathrm{al}^{3}$ reported diffuse proximal tubular injury with brush border loss, vacuolar degeneration, necrosis and prominent erythrocyte aggregates without any platelet/ fibrinoid material in the occluded microvasculature. No evidence of interstitial inflammation/hemorrhage or vasculitis was noted. They also reported groups of particles resembling coronavirus in the tubular epithelium and podocytes and up regulation of SARS-CoV-2 receptors (such as ACE2) in these patients. These findings suggested that kidneys may be affected even in mild forms of COVID-19 infection. In addition to the unambiguous cytotoxicity of COVID-19, abnormal coagulation, systemic hypoxia, and rhabdomyolysis can also contribute to the acute renal injury.

Renal artery thrombosis showed a reported prevalence of $0.02 / 1,000$ in emergency department patients, whereas it showed a prevalence of $14 / 1,000$ in an autopsy series of $14,000 .^{8}$ Atrial fibrillation is the most common cause of renal artery thrombosis, followed by cholesterol-based emboli. ${ }^{8}$ In patients with a history of atrial fibrillation, peripheral thromboembolic events involved arteries of the extremities in $61 \%$ of cases, whereas renal arteries were affected in only $2 \%$ of cases. ${ }^{8}$

Renal infarction is a relatively rare entity and has a reported incidence of $0.1-1.4 \% .^{2}$ Clinical diagnosis of renal infarction is challenging because of its low incidence and nonspecific clinical features. ${ }^{2}$ Imaging plays a vital role in its diagnosis, with contrast-enhanced CT of the abdomen being the imaging modality of choice, although the gold standard of imaging is conventional renal angiography. ${ }^{2}$ Transportation of COVID-19 patients, particularly intubated ICU patients, and IV contrast issues in patients with renal failure are the 
major limitation factors of a contrast-enhanced CT scan. Additionally, a CT cannot diagnose micro vascular thrombi. ${ }^{1,2}$ Renal ultrasound examination is of limited value due to its lower sensitivity. ${ }^{1}$

Coronavirus disease-19-infected patients with renal involvement often have renal function abnormalities. Renal injury was observed in approximately $80 \%$ cases of COVID-19 pneumonia. ${ }^{8}$ The pathophysiology of acute kidney injury in COVID-19 infection is still not completely understood but may be due to the direct cytotoxic effects of the virus on the tubular and endothelial cells of the kidney, indirect damage (by virus-induced cytokine release), or renal hypoperfusion. ${ }^{1}$

Based on the initial presentation (+ COVID-19 PCR without any symptoms), our case was defined as a mild COVID-19 infection and was managed with home isolation only. Two weeks later, he presented to the emergency department with acute left flank pain. Renal pathology was not expected clinically and was just an incidental diagnosis based on the CT of the abdomen carried out because of his complaint. Except for the left flank pain, there were no other specific clinical findings to suspect a renal abnormality. The CT findings were suggestive of multiple acute left renal infarctions (with patent main renal vessels), which along with the mildly elevated D-dimer level, were thought to be attributed to the COVID-19-induced hypercoagulopathy or endotheliopathy at the microvasculature level.

To date, few case reports of radiological findings of renal infarctions had been observed that were secondary to the marked hypercoagulopathy observed in severe COVID-19 infections. ${ }^{1,2,4,6,8,10}$ However, as in our case, a few case reports of renal infarctions in mild COVID-19 infections had also been reported in the literature. ${ }^{6,7,9}$

At present, the American Society of Hematology recommended prophylactic LMWH for all hospitalized patients with therapeutic doses being reserved for seriously infected intubated patients with clinical suspicion of pulmonary embolism, deep venous thrombosis, and for patients with high D-dimer or fibrinogen levels. ${ }^{7}$

In conclusion, the thromboembolic consequences of COVID-19 can occasionally result in rare complications, such as renal infarction. Based on our case, we recommend that clinicians be vigilant of abdominal symptoms of COVID-19 infection, even in mild/ asymptomatic cases and suspect COVID-19 infection in patients with unexpected thrombotic events in the current era of the COVID-19 pandemics. Physicians should have a low threshold for requesting abdominal imaging even in cases of mild COVID-19 infection with minor abdominal complaints. Renal infarction should be included as a possible differential diagnosis in COVID-19-infected patients who present with flank pain, acute kidney injury, or new onset proteinuria. We also suggest large-scale clinical trials targeting the prevention and management of such complications of COVID-19 infection.

Acknowledgment. The authors gratefully acknowledge Dr. Syed H. Hassan (consultant radiologist) for reviewing the CT images. The authors would also like to thank the Falcon Scientific Editing (www.falconediting.com) for English language editing.

\section{References}

1. Post A, den Deurwaarder ESG, Bakker SJL, de Haas RJ, van Meurs M, Gansevoort RT, et al. Kidney infarction in patients with COVID-19. Am J Kidney Dis 2020; 76: 431-435.

2. Mukherjee A, Ghosh R, Furment MM. Case report: COVID-19 associated renal infarction and ascending aortic thrombosis. Am J Trop Med Hyg 2020; 103: 1989-1992.

3. Su H, Yang M, Wan C, Yi LX, Tang F, Zhu HY, et al. Renal histopathological analysis of 26 postmortem findings of patients with COVID-19 in China. Kidney Int 2020; 98: 219-227.

4. Xu JJ, Samaha D, Mondhe S, Massicotte-Azarniouch D, Knoll G, Ruzicka M. Renal infarct in a COVID-19-positive kidney-pancreas transplant recipient. Am J Transplant 2020; 20: 3221-3224.

5. Connors JM, Levy JH. COVID-19 and its implications for thrombosis and anticoagulation. Blood 2020; 135: 2033-2040.

6. Basara Akin I, Altay C, Eren Kutsoylu O, Secil M. Possible radiologic renal signs of COVID-19. Abdom Radiol (NY) 2021; 46: 692-695.

7. Kundal S, Emeasoba EU, Harris C, Randhawa G, Astashkevich M. Aortic thrombosis and renal infarction in a young female with patent foramen ovale and COVID-19 antibody. Clin Case Rep 2020; 9: 345-349.

8. El Shamy O, Munoz-Casablanca N, Coca S, Sharma S, Lookstein R, Uribarri J. Bilateral renal artery thrombosis in a patient with COVID-19. Kidney Med 2021; 3: 116-119.

9. Varner KB, Cox EJ. COVID-19 as the cause of thrombosis: recognising COVID-19 infection in apparently asymptomatic patients. BMJ Case Rep 2021; 14: e241027.

10. Ammous A, Ghaffar MA, El-Charabaty E, El-Sayegh S. Renal infarction in COVID-19 patient. J Nephrol 2021; 34: 267-268. 